\title{
Analysis on the Law of Chinese Medicine Use of Ulcerative Colitis and TCM Syndrome Differentiation and Treatment
}

\author{
Jiaojiao Zhang, Heng Mu, Xiaogang Wang, Xingxing Yu, Fan Zhang, Yi Li* \\ Shaanxi University of Traditional Chinese Medicine, Xianyang 712046, Shaanxi Province, China
}

Funding: Based on Th17/Treg immune network, the mechanism of action and clinical efficacy evaluation of Fuyang Huoxue Jiedu Decoction against recurrence of ulcerative colitis were studied

\begin{abstract}
Ulcerative colitis is a kind of inflammatory bowel disease. The disease is slow and easy to relapse. Western medicine treatment mainly focuses on symptomatic treatment, which has certain side effects on liver and kidney function. Ulcerative colitis has unique advantages in disease, so by studying a large amount of information, mainly from the law of traditional Chinese medicine use of UC, and a brief summary of the treatment of TCM syndromes, this article provides ideas and basis for clinical diagnosis and treatment of the disease.
\end{abstract}

Key words: Ulcerative colitis; Law of traditional Chinese medicine; Syndrome differentiation and treatment

Publication date: May, 2021; Publication online: 31 May, 2021

*Corresponding author: Yi Li, 253197724@qq.com

Youlcerative colitis (UC) is a common recurrent disease of the digestive system, which is characterized by inflammation and rectal mucosa, mainly abdominals, abdominals, and abdominal pain. This disease can be seen at any age.In recent decades, the incident of this disease in China has risen sharply ${ }^{[1]}$.

The ecology and pathologies of Western medicine are still unclear, but modern medicine believes that genetic susceptible, environmental factors and immune factors are important factors in the West of UC. Some scholars also pointed out that the imbalance of the intestinal flora and it's metabolism can trigger a series of inflammatory reactions, which will lead to immune disorders and induce disorders.

\section{Understanding of traditional Chinese medicine}

"I must read medical":"The syndrome of dysentery has multiple splen and kidneys.Patients with spelt ill, those with kidneys are deeply ill, and those with no chronic dysentery without kidney damage."Kidney-related, congenital inefficient, acquired daytrophy, six evils, abnormal daily life, etc.Cause the intestinal dysfunctional damage, the ability to decompose, the poor circulation of the qi and blood, and damage to lipid membranes and blood network.

\section{Analysis of the law of the use of traditional}

\section{Chinese medicine}

\subsection{Single medicine}

An analysis of the law of traditional Chinese medicine for UC based on data mining concluded that the most commonly used single Chinese medicines for the clinical treatment of UC are licorice, Atractylodes, Coptis, Codonopsis, etc., mainly tonic drugs, heat-clearing drugs, and Qi regulating drugs. The first two have the highest frequency of medication. Ingredients are calm in nature, sweet in taste, effective in benefiting qi, expelling phlegm, relieving pain, and recognizing various medicines. It can effectively relieve cough, dyspnea, cramps and pain. Atractylodes macrocephala tastes bitter, sweet, warm in nature, functions to invigorate the skin and qi, dry dampness, 
diuresis, antiperspirant, and antipers. Studies have shown that the extract of Atractylodes macrocephala has liver protection, znti-inflammato ,anti-thranalytic effects.

2.2 Association rules, drug properties, meridians, and efficacy

According to the analysis of UC medication rules, the most frequently occurring oral related drugs are Atracted macrocephala-Poria, Atractylodes macrocephala-Licorice, Atractylodes macrocephala-Citrus tangerine peel. The most commonly used clinically related drugs are qi and spelt-invigorating drugs. Cyprusis and dampness drugs, Qi regulation drugs, of which the qi tonics and spedes insuring drugs are dominant. It can be found that the key to the treatment of UC lies in the treatment of the splash, and the spoken tonic is the treatment method. From the analysis of nature and taste of traditional Chinese medicine, the warm, cold, and bitter taste are the main ones.In the meridian, the spleen and stomach meridians and the liver meridian are the highest, followed by the lung meridian; in terms of efficacy, the most frequently appearing are the tonic drugs, heat-clearing drugs, and medicine.

\section{TCM syndrome have differentiation and}

\section{treatment}

Traditional medicine has unique advantages in the treatment of this disease. Combining syndrome differentiation and treatment, using Chinese medicine, acupuncture, enema and other therapies, the prescription is flexible and flexible, with the addition and subtraction of the syndrome, the effect is wide, the adverse reaction is small, and the clinical symptoms are effectively relieved.

\subsection{Inner proof of dampness and heat}

This syndrome often manifests as tenesmus with diarrhea, red and white pus and blood in the stool, short red urine and other symptoms. Peony Decoction has the effects of regulating qi and blood, clearing heat and removing dampness. Scutellaria baicalensis and coptidis are selected as the king of drug in the prescription to relieve the damp heat in the intestine. Re-use of peony, restrains yin and nourishes blood, relieves emergency pain, angelica nourishes blood and nourishes blood, the combination of the two medicines has the effect of invigorating blood and regulating blood, effectively alleviating the damage of yin and blood caused by damp-heat burning and intestinal collateral damage. Modern pharmacological studies have shown that it can act on multiple cellular components to produce different biological functions, and has the characteristics of "multi-component-multi-target-multi-pathway". Xu Min ${ }^{[2]}$ found that Shaoyao Decoction regulates the excessive activation of the TLR4/NF- $\mathrm{BB}$ pathway, which in turn affects the expression of IL-6 and other immune networks, and plays a key role in the development of UC.

\subsection{Deficiency of spleen and stomach qi}

Chronic disease is due to spleen deficiency and stomach weakness, loss of transport and transformation, accumulation of water and dampness, and retention of intestinal collaterals, so the disease develops. UC is a syndrome of spleen-stomach-qi deficiency and is mostly manifested by symptoms of low digestive function such as fullness of the abdomen, loose stools and so on. Shenling Baizhu Powder is composed of ginseng, atractylodes macrocephala, poria, yam, etc. The first three are the monarch medicines matched with medicines such as antidiarrheal, stomach, and lungs and qi, that play the effects of invigorating qi, strengthening the spleen, exuding dampness and stopping diarrhea. Modern pharmacological studies have shown that this formula has antidiarrheal and analgesic effects, can regulate immune disorders, and promote the repair of damaged intestinal mucosa. Li Zihui ${ }^{[3]}$ proved through animal experiments that Shenlingbaizhu Powder regulates the expression of $\mathrm{NF}-\kappa \mathrm{B}$ and other upstream kinases in the rat colon and inhibits the release of downstream related inflammatory factors, thereby reducing intestinal inflammation and alleviating intestinal mucosal damage.

\subsection{Liver depression and spleen deficiency syndrome}

The main symptoms of UC with liver depression and spleen deficiency include bowel sounds and abdominal pain, diarrhea, tightness in the chest and hypothermia, and narrow pulse. The main prescription for pain and diarrhea contains four herbs of Atractylodes macrocephala, peony root, dried tangerine peel and wind-proof. The monarch drug Atractylodes macrocephala can invigorate the spleen and dry dampness to cure spleen deficiency; the minister drug white peony can relieve pain and relieve pain, restrain the yin and nourish blood; adjuvant it is used to regulate qi and dry dampness. Tangerine peel and Xin San are the anti-wind for soothing the liver, combined with surgery and peony, and strengthen the power of dampness to help stop diarrhea. The four medicines are shared to invigorate the spleen and 
overcome dampness and relieve diarrhea, sooth the liver and regulate qi and relieve pain. A large number of studies have confirmed that Tongxie Yaofang can effectively relieve the symptoms of diarrhea and abdominal pain, and its mechanism may be to inhibit excessive immune response and prevent further damage to the intestinal mucosa.

\subsection{Spleen and kidney yang deficiency syndrome}

The main manifestations of this syndrome are pain in the umbilical abdomen before dawn, the grains are not melted, the waist and knees are sore, and the pulse is sinking. The spleen and kidney are the foundation of the heavens, the spleen governs the promotion of clearness, transport and transformation, and the kidney governs the warmth of the five internal organs. The two influence each other and are mutually cause and effect. If the spleen and kidney are insufficiency, the body's temperature is dereliction of duty, loss of transport and transformation, and water retention. Therefore, it is diarrhea. Fuzi Lizhong decoction, the prescription of Fuzi Daxin and Dare, can warm the kidney yang, so the UC of spleen and kidney yang deficiency is commonly used in this prescription. Sishen Pill is another classic prescription for the treatment of this syndrome. The prescription contains psoralen, evodia, schisandra, and nutmeg, which can exert curative effects by interfering with multiple systems such as circulation, digestion, and immunity.

\section{Summary}

In the past few decades, the incidence of UC has generally increased, which has seriously affected people's quality of life. My country has abundant resources of traditional
Chinese medicine. Chinese medicine not only has antiviral effects, but the problem of resistance of traditional Chinese medicine is not obvious. Therefore, it is necessary to strengthen the characteristics of traditional Chinese medicine therapy and traditional Chinese medicine pharmacology research, and screen special Chinese medicines and special prescriptions to promote the development of UC. The standardization of TCM diagnosis and treatment was then promoted to the international community. In the process of clinical diagnosis and treatment, we should also fully grasp the law of disease incidence, build a more comprehensive diagnosis and treatment framework, and strive to overcome this medical problem as soon as possible.

\section{References}

[1] Cui GL, and Yuan A. A systematic review of epidemiology and risk factors associated with chinese inflammatory bowel disease $[\mathrm{J}]$. Frontiers in Medicine, 2018, 5: 183.

[2] Xu M, Wang FY, Zhao DS, et al. Regulation of Shaoyao Decoction on the expression of TLR4, NF- $\kappa B$ p65 and IL-6 in rats with damp-heat intrinsic ulcerative colitis $[\mathrm{J}]$. Chinese Journal of Experimental Traditional Chinese Medicine, 2020,26(14):53-58.

[3] Li ZH, Cai RL, Sun J, et al. Effects of Shenlingbaizhu Powder on the expression of NF- $\kappa \mathrm{B} p 65, \mathrm{I} \kappa \mathrm{B} \alpha, \mathrm{I} \kappa \mathrm{K} \beta$ protein and mRNA in colon tissue of rats with ulcerative colitis of spleen deficiency and dampness type [J]. China Experimental Formulae Journal, 2020, 26(19): 108-113. 